\title{
Structural transition in Fe ultrathin epitaxial films grown on Ni(111)
}

\author{
G. C. Gazzadi, ${ }^{1, *}$ F. Bruno, ${ }^{2}$ R. Capelli, ${ }^{1}$ L. Pasquali, ${ }^{1}$ and S. Nannarone ${ }^{1}$ \\ ${ }^{1}$ INFM and Dipartimento di Fisica, Università di Modena e Reggio Emilia, Via G. Campi 213/a, 41100 Modena, Italy \\ ${ }^{2}$ Laboratorio TASC-INFM, Area Science Park, S.S.14 Km 163.5, I-34012 Basovizza, Trieste, Italy
}

(Received 23 April 2001; revised manuscript received 17 October 2001; published 15 May 2002)

\begin{abstract}
A structural study of Fe ultrathin epitaxial films, grown at room temperature on $\mathrm{Ni}(111)$, has been performed in the 1.5-18 ML coverage range by angle-scanned photoelectron diffraction. Both backscattering and forward-scattering energy regimes have been employed, in order to enhance the structural sensitivity at lower and higher film thicknesses, respectively. Modeling of the experimental data has been performed with multiple scattering calculations. We found indications that Fe atoms in the first layer occupy fcc hollow sites and stack with a pseudomorphic fcc structure up to $2 \mathrm{ML}$. Concerning the growth mode at these early stages, data suggest that a good substrate wetting and a sharp Fe/Ni interface take place. Between 3 and $6 \mathrm{ML}$, transition to a bcc(110) phase develops. By quantitative $R$-factor analysis, we found that Nishiyama-Wassermann (NW) in-plane orientation of the bcc(110) cell $\left(\langle 001\rangle_{\mathrm{bcc}} \|\langle 1 \overline{1} 0\rangle_{\mathrm{fcc}}\right)$ is favored over the Kurdjumov-Sachs $\left(\langle 1 \overline{1} 1\rangle_{\mathrm{bcc}} \|\langle 1 \overline{1} 0\rangle_{\mathrm{fcc}}\right)$ orientation. The best-fit vertical interlayer distance between bcc(110) planes is $d_{\mathrm{NW}}$ $=2.11 \AA(+3.9 \%$ expansion $)$ at $6 \mathrm{ML}$ and relaxes to $d_{\mathrm{NW}}=2.05 \AA(+1.0 \%)$ at $18 \mathrm{ML}$, in agreement with the angular shift observed for the forward-focusing features. In the same coverage range, the angle between bcc(110) surface basis vectors changes from $67.7^{\circ}$ to $69.0^{\circ}$, corresponding to $-1.7 \%$ and $-1.0 \%$ contractions of the surface cell area, respectively.
\end{abstract}

DOI: 10.1103/PhysRevB.65.205417

PACS number(s): 68.55.Jk, 61.14.Qp

\section{INTRODUCTION}

Since the theoretical prediction that $3 d$ transition metals would show a magnetovolume instability, ${ }^{1-3}$ growth of ultrathin epitaxial films of such elements has attracted increasing interest in surface science. Calculations showed that, by stabilizing these elements in structural phases different from their natural ones, peculiar and generally enhanced magnetic properties could be obtained. In the case of $\mathrm{Fe}$, such a behavior was shown for the low-temperature $\gamma$ phase (fcc), which is naturally stable only at high temperatures for the bulk, but can exist at room temperature (RT) in thin epitaxial films grown on suitable fcc substrates. The necessary requirement for a close lattice match between substrate and deposit and for a magnetic study of the thin film usually led to choose nonmagnetic fcc templates like $\mathrm{Cu}^{4,5}$ Nickel is suitable as well, as far as the lattice mismatch is concerned $(+2 \%$, referred to the RT lattice parameter of fcc Fe, extrapolated from the high-temperature phase ${ }^{6}$ ), but it is ferromagnetic and this may hinder the study of the magnetic properties of the Fe film. On the other hand, employing element specific magnetic probes, ${ }^{7}$ it can be considered as an opportunity to explore the influence of a magnetic substrate on the magnetism of the overlayer.

Besides magnetism, growth of $\mathrm{Fe}$ on $\mathrm{Ni}(111)$, and generally the heteroepitaxy of bcc/fcc metals, represents an interesting topic from the structural point of view, which has received considerable theoretical attention. ${ }^{8-10}$ When $\mathrm{Fe}$ is grown on fcc(111) substrates, the evolution of the film structure vs thickness is expected to show a transition from a pseudomorphic fcc to a bcc phase, which resembles, in a reversed way, the bcc-to-fcc martensitic transition occurring in bulk Fe vs temperature. This structural transition develops with fcc(111) planes growing parallel to bcc(110) ones at the interface between the two phases. Therefore, the use of (111)-oriented fcc substrates is expected to be particularly suited in studying the transition. Another question regards the in-plane orientation between bcc and fcc surface cells, which may follow either Nishiyama-Wassermann (NW) $\quad\left(\langle 001\rangle_{\mathrm{bcc}} \|\langle 1 \overline{1} 0\rangle_{\mathrm{fcc}}\right) \quad$ or Kurdjumov-Sachs $\quad(\mathrm{KS})$ $\left(\langle 1 \overline{1} 1\rangle_{\mathrm{bcc}} \|\langle 1 \overline{1} 0\rangle_{\mathrm{fcc}}\right)$ models, depending on the ratio of unit vectors $\left(r=a_{\mathrm{bcc}} / a_{\mathrm{fcc}}\right)$ and on the layer-substrate interaction strength. ${ }^{9}$ The $\mathrm{Fe} / \mathrm{Ni}$ case is of particular interest because it is predicted to have a critical dependence on these parameters.

A theoretical structural and magnetic study by $\mathrm{Wu}$ and Freeman ${ }^{11}$ investigated the $\mathrm{Fe}$ behavior within the first monolayer (ML), finding that the favorite adsorption site on $\mathrm{Ni}(111)$ at $0 \mathrm{~K}$ was the hcp hollow and not the fcc one, with a downward relaxation of the $\mathrm{Fe} / \mathrm{Ni}$ interlayer distance and a decrease of the Fe magnetic moment. To address experimentally the question of the adsorption site, Theobald et al. ${ }^{12}$ performed energy-scanned photoelectron diffraction (PD) on a $0.6-\mathrm{ML}$ film at $130 \mathrm{~K}$, finding $\mathrm{Fe}$ atoms in the fcc hollow site, with an Fe-Ni interlayer distance of $2.00 \AA$ A. Other experimental investigations ${ }^{13,14}$ have been performed using a few monolayers of Ni deposited on W(110) as substrates, in order to obtain fcc(111) Ni templates with reduced magnetic influence on the Fe films. Fcc Fe has been observed up to 3-4 ML, followed by a complex transition to a bcc(110) structure, involving both KS and NW orientations. However, the complexity of this structural evolution might have been influenced by the nonideality of the $\mathrm{Ni}(111)$ substrates, which show in-plane distortions and multidomains growth on $\mathrm{W}(110)$. Therefore, Fe growth on $\mathrm{Ni}(111)$ single-crystal substrates has been clarified only within the first ML and knowledge of the structural evolution at higher coverage still lacks. We have already investigated in detail the growth of epitaxial Fe films on $\mathrm{Ni}(001),{ }^{15-17}$ focusing particularly on the structural aspects, because of the crucial role played by these on 
the magnetic properties of such systems. Magnetism of $\mathrm{Ni} /$ $\mathrm{Fe} / \mathrm{Ni}(111)$ trilayers and $\left(\mathrm{Fe}_{6} \AA / \mathrm{Ni}_{24} \AA\right)_{10} / \mathrm{Ni}(111)$ multilayers has been studied by Mössbauer spectroscopy ${ }^{18}$ and by absorption and reflectivity measurements, ${ }^{7}$ respectively, finding an enhancement of the total magnetic moment of the $\mathrm{Fe}$ atoms at the interface. In a recent work, ${ }^{19}$ we studied the electronic properties of $\mathrm{Fe} / \mathrm{Ni}(111)$ films by electron energy loss spectroscopy at the valence band and at the Fe $L_{2,3}$ edges, following the structural evolution by low-energy electron diffraction (LEED). We were able to correlate changes in the $3 d$ band region with the structure evolution, identifying a critical thickness of $4 \mathrm{ML}$ for the fcc-to-bcc transition.

In the present study we apply angle-scanned PD (Ref. 20) by means of synchrotron radiation, to follow the structural evolution of $\mathrm{Fe} / \mathrm{Ni}(111)$ films in the 1.5-18 ML coverage range. The medium-to-high coverage region has been studied in the forward-scattering energy regime, which enables a direct link between structural changes and the angular position and shape of the forward-focusing features. The lowest coverage has been investigated in the low-energy backscattering regime to enhance both the surface and the back-bond sensitivity. To extract a quantitative information, multiple scattering (MS) calculations and $R$-factor analysis have been performed. The paper is organized as follows. In Sec. II, details on the experiment and on the data modeling are given. The results are presented in Sec. III, divided into three sections: Secs. III A and III B present the experimental results for the clean substrate and for the Fe films, respectively. Section III C presents data modeling by MS calculations divided into two sections: Sec. IIIC 1 for the low coverage and Sec. IIIC 2 for the medium-to-high coverage. Results are discussed in Sec. IV and, finally, in Sec. V, conclusions are presented.

\section{EXPERIMENT AND DATA MODELING}

Growth and structural characterization of the Fe films were done in situ at the ultrahigh vacuum end station of beam line 7.2 (INFM, ALOISA), ${ }^{21}$ located at the ELETTRA Synchrotron (Trieste, Italy). The $\mathrm{Ni}(111)$ surface was prepared by sputtering and annealing cycles by means of $1-\mathrm{keV}$ Ar ions and $600^{\circ} \mathrm{C}$ temperature. The surface cleanliness and local order were checked by photoelectron spectroscopy and PD measurements, respectively. The base pressure was 2 $\times 10^{-10}$ Torr, rising to $8 \times 10^{-10}$ Torr during evaporations. Fe was evaporated by means of electron-bombardment cells from $99.999 \%$ purity Fe rods. The deposition rate was monitored by means of a quartz microbalance and calibrated by fitting $\mathrm{Fe} 2 p / \mathrm{Ni} 2 p$ intensity ratio data with an exponential attenuation model of the secondary electron intensity, using inelastic mean free paths (IMFP's) taken from the literature. ${ }^{22}$ The growth rate was $0.40 \pm 0.06 \mathrm{ML} / \mathrm{min}$, assuming a ML thickness of $2.03 \AA$, the Ni interlayer distance along the [111] direction. The degree of contamination after evaporation was checked by photoemission from $\mathrm{C} 1 s$, O $1 s$, and Fe $3 p$ core levels, for a 8-ML-thick film (20 min evaporation). Taking into account the different photoemission cross sections and IMFP's, we estimated an amount of carbon and oxygen of the order of 9 at. $\%$ and 4 at. \%, re- spectively, assuming the two species uniformly distributed into the Fe film.

PD measurements consisted of measuring intensity angular distributions (IAD's) of the photoelectrons, by scanning an hemispherical analyzer ( $1^{\circ}$ angular resolution) over the polar takeoff angle $\theta$ in the planes defined by the surface normal and by the three nonequivalent azimuthal directions of the $\mathrm{Ni}(111)$ surface: the $[1 \overline{2} 1]_{\mathrm{fcc}}\left(\phi_{\mathrm{fcc}}=0^{\circ}\right)$, the $[1 \overline{1} 0]_{\mathrm{fcc}}$ $\left(\phi_{\mathrm{fcc}}=30^{\circ}\right)$, and the $[2 \overline{1}]_{\mathrm{fcc}}\left(\phi_{\mathrm{fcc}}=60^{\circ}\right)$ directions. Polar angles were varied by $\Delta \theta=1^{\circ}$ and measured with respect to the sample normal. In forward-scattering regime measurements, Fe $2 p$ and Ni $2 p$ photoelectrons were collected with a kinetic energy $E_{k}=480 \mathrm{eV}$. In backscattering regime measurements, the intensity pattern of low-energy $\left(E_{k}\right.$ $=120 \mathrm{eV}) \mathrm{Fe} 2 p$ photoelectrons was taken by measuring IAD's over a $120^{\circ}$ azimuthal range, starting from $\phi_{\mathrm{fcc}}=0^{\circ}$ in steps of $\Delta \theta=\Delta \phi=2^{\circ}$.

Data modeling was performed with the MSCD $\operatorname{code},^{23}$ treating MS in the Rehr-Albers approximation. We considered MS events up to the fifth order. Phase shifts were calculated up to angular momentum number $l=15$, based on muffin-tin potentials taken from Ref. 24. Atomic clusters were defined by the radius $r$ and the depth $h$ of a semiellipsoid, having the circular basis on the top layer and the vertex on the bottom one. In all calculations, $r$ was set equal to 10 $\AA$ and the depth $h$ was varied according to the convolution of the film thickness and the escape depth of the photoelectrons. For the low coverage data (1.5 ML), clusters containing three $\mathrm{Ni}$ layers and either one or two $\mathrm{Fe}$ layers on top were employed. At higher coverages, i.e., for 6-, 9-, and 18-ML data, a depth $h$ large as $9 \AA$ (i.e., five Fe layers), of the order of the IMFP of Fe $2 p$ photoelectrons, was found sufficient to reproduce all the measured features. The treatment of experimental and calculated IAD curves, before performing $R$-factor calculations, followed the procedure reported in a previous paper. ${ }^{15}$

\section{RESULTS}

\section{A. $\mathrm{Ni}(111)$ clean surface}

The Ni $2 p$ IAD's, collected along the $[1 \overline{2} 1]_{\mathrm{fcc}},[1 \overline{1} 0]_{\mathrm{fcc}}$, and $[2 \overline{1} \overline{1}]_{\text {fcc }}$ azimuths on the clean $\mathrm{Ni}(111)$ surface, are shown in Fig. 1. We notice, at first, that a photoelectron kinetic energy of $480 \mathrm{eV}$ is high enough to give a remarkable forward-focusing effect; therefore, the attribution of the main features observed in each IAD becomes straightforward. Data measured along the $[1 \overline{2} 1]_{\mathrm{fcc}}$ azimuth are characterized by a large peak around $35^{\circ}$, corresponding to the forward focusing of electrons along the close-packed [011] direction. Along the $[1 \overline{1} 0]_{\mathrm{fcc}}$ azimuth, the IAD displays two main offnormal features at $22.2^{\circ}$ and $39.2^{\circ}$ corresponding to forward focusing along the [132] and [021] atomic directions, respectively. The IAD collected along the $[2 \overline{1} \overline{1}]_{\text {fcc }}$ azimuth shows the strongest features at $20^{\circ}$ and $55^{\circ}$, related to the [112] and [001] directions, respectively. Measurements along the $[1 \overline{2} 1]_{\mathrm{fcc}}$ and $[2 \overline{1} \overline{1}]_{\mathrm{fcc}}$ azimuths are scanning the same family of atomic planes $[(20 \overline{2})$ and $(0 \overline{2} 2)$, respectively] in two 


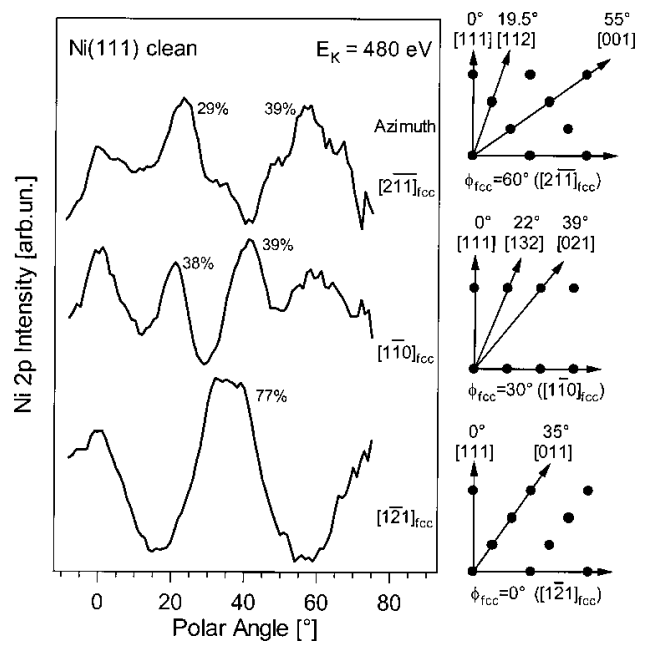

FIG. 1. Intensity angular distributions (IAD's) of Ni $2 p$ photoelectrons collected at $E_{K}=480 \mathrm{eV}$ along the $[1 \overline{2} 1]_{\mathrm{fcc}},[1 \overline{1} 0]_{\mathrm{fcc}}$, and $[2 \overline{1} \overline{1}]_{\text {fcc }}$ azimuths on the clean $\mathrm{Ni}(111)$ surface. A crosssectional view of atomic planes scanned along each azimuth is sketched together with close-packed atomic directions.

opposite directions with respect to the sample normal. The lack of mirror symmetry with respect to the [111] axis (see the cross sections of atomic planes sketched in Fig. 1) is reflected by the markedly asymmetric shape of the $0^{\circ}$ feature in the two IAD's. In Fig. 1, the intensity anisotropy $[A$ $\left.=\left(I_{\max }-I_{\text {min }}\right) / I_{\text {mean }}\right]$ values are reported beside each forwardfocusing feature. The anisotropy of a forward feature is a figure of the strength of the electron focusing along the atomic chain. Its value is related to the atomic arrangement within the chain and, roughly, it is higher for closer packing. We used it as a parameter to check the quality of the local crystalline order during thin-film growth and substrate preparation. We notice the largest value of $77 \%$, associated with the closest-packed chain, the [011] direction.

\section{B. $\mathrm{Fe} / \mathrm{Ni}(111)$ films}

Fe $2 p$ IAD's, measured along the $[1 \overline{2} 1]_{\mathrm{fcc}},[1 \overline{1} 0]_{\mathrm{fcc}}$, and $[2 \overline{1} \overline{1}]_{\mathrm{fcc}}$ azimuths at increasing Fe coverage, are shown in Fig. 2 together with the corresponding Ni $2 p$ IAD, collected on the clean substrate. At 1.5 ML, no clear feature in the three Fe IAD's appears as a function of the polar angle, except, perhaps, for the curves along the $[1 \overline{2} 1]_{\mathrm{fcc}}$ and $[2 \overline{1} \overline{1}]_{\text {fcc }}$ azimuths, where a very weak bump at large polar angles can be observed. As the Fe coverage increases, welldefined structures grow up. Along the $[1 \overline{2} 1]_{\mathrm{fcc}}$ azimuth, starting from $3 \mathrm{ML}$, two features develop at $0^{\circ}$ and $47^{\circ}$, the latter showing a small shoulder at $60^{\circ}$, at higher coverages. Along the $[1 \overline{1} 0]_{\mathrm{fcc}}$ azimuth, two broad features develop around $0^{\circ}$ and $32^{\circ}$, becoming sharper and more defined with coverage. At $6 \mathrm{ML}$ a third, weaker, peak appears at $57^{\circ}$. Along the $[2 \overline{1} \overline{1}]_{\mathrm{fcc}}$ azimuth the main off-normal feature grows up at $55^{\circ}$, starting as a broad peak and turning into a doublet at $50^{\circ}$ and $60^{\circ}$ at high coverage. Increasing the coverage, another small feature at $27^{\circ}$ also appears.
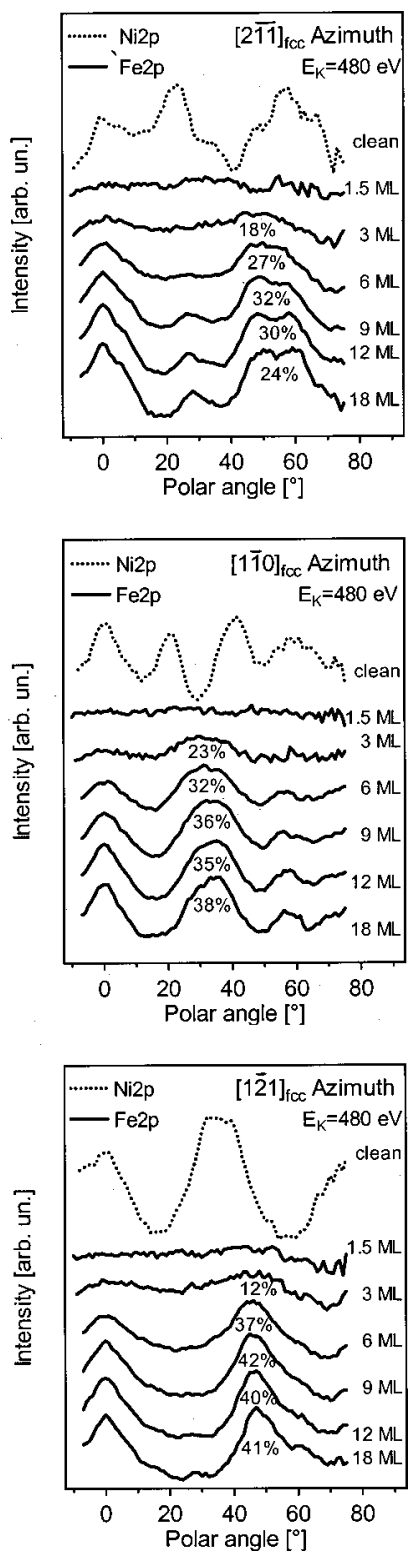

FIG. 2. Intensity angular distributions (IAD's) of Fe $2 p$ photoelectrons collected at $E_{K}=480 \mathrm{eV}$ along the $[1 \overline{2} 1]_{\mathrm{fcc}},[1 \overline{1} 0]_{\mathrm{fcc}}$, and $[2 \overline{1} \overline{1}]_{\text {fcc }}$ azimuths on Fe films of increasing thickness. Ni $2 p$ IAD's for the clean substrate are also displayed.

All the off-normal forward peaks undergo a detectable shift towards higher polar angles as the coverage increases, indicating that a downward relaxation in the vertical interlayer distance occurs as the film grows. A final shift of about $2.5^{\circ}$, similar for all the peaks, was measured. For what concerns the intensity anisotropy, it progressively increases up to $9 \mathrm{ML}$ and then stabilizes around the maximum value or slightly decreases, as already reported during thin-film epitaxial growth.

The substantial flatness of the 1.5-ML IAD's hinders the assignment of $\mathrm{Fe}$ atomic structure at low coverage but allows some considerations on the morphology of the $\mathrm{Fe} / \mathrm{Ni}$ interface. It is a clear indication that the interface is sharp, with weak $\mathrm{Fe} / \mathrm{Ni}$ intermixing and absence of thick islands. In fact, the picture suggested by the data is the one that minimizes 
a)

$$
\begin{array}{cc}
\text { Nishiyama-Wassermann (NW) } & \text { Kurdjumov-Sachs (KS) } \\
<001>_{\text {bcc }} \|<1 \overline{1} 0>_{\text {fcc }} & <1 \overline{1} 1>\text { bcc } \|<1 \overline{1} 0>_{\text {fcc }}
\end{array}
$$
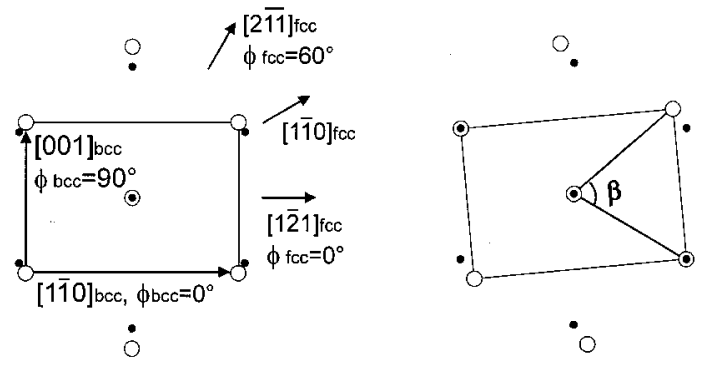

b)

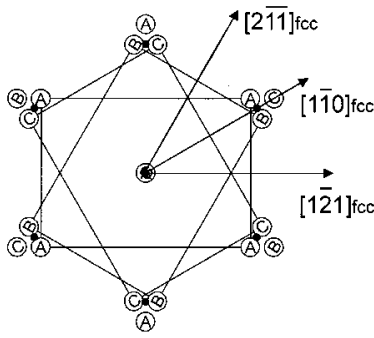

3 NW domains

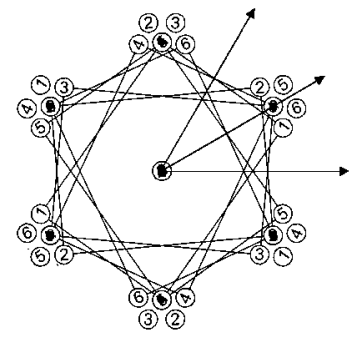

$6 \mathrm{KS}$ domains

FIG. 3. (a) In-plane orientation relationship between bcc(110) (open circles) and fcc(111) (closed circles) surface cells in the NW and KS models. (b) The three NW domains are indicated by letters and the six KS domains by numbers.

the number of forward-focusing events at that coverage. Namely, the first $\mathrm{Fe}$ layer uniformly wets the substrate and half of the second layer adds on top of it, producing the few focusing events responsible for the weak modulations at large polar angles. As soon as some forward features are detectable, i.e., from $3 \mathrm{ML}$ on, it is evident that they differ in number and angular position from those of the substrate at the corresponding azimuth; therefore, we have to conclude that the Fe film is not pseudomorphic with the substrate above this coverage and a new structural phase is growing. We also notice that the key features characterizing the high coverage IAD's are already defined at $3 \mathrm{ML}$, and only rise in intensity, undergoing small angular shifts, as the coverage increases. This also indicates that the film structure is already defined at this coverage and no abrupt phase transition occurs above. This behavior is of pertinent interest to the determination of the Fe structural phase below $3 \mathrm{ML}$, in particular, to establish whether it grows in the new phase already from the beginning or it is pseudomorphic with the substrate. As previously mentioned, from the literature it is known that the structural evolution of bcc metals grown on fcc(111) substrates leads to the formation of a bcc(110) phase with two possible in-plane orientation relationships, relative to the underlying fcc lattice: the Nishiyama-Wassermann (NW) $\left(\langle 001\rangle_{\text {bcc }} \|\langle 1 \overline{1} 0\rangle_{\text {fcc }}\right)$ and the Kurdjumov-Sachs (KS) $\left(\langle 1 \overline{1} 1\rangle_{\mathrm{bcc}} \|\langle 1 \overline{1} 0\rangle_{\mathrm{fcc}}\right)$, which are schematically represented in Fig. 3(a). The threefold symmetry of the fcc(111) surface generates three equivalent domains in the $\mathrm{NW}$ orientation

and six in the KS one. In Fig. 3(b), the possible NW and KS domains are superimposed to the substrate surface lattice, using letters $(A-C)$ and numbers $(1-6)$ as atomic site markers, respectively. A qualitative interpretation of the high coverage data can be given by looking for a correspondence between the angular position of the measured features and the close-packed atomic rows that are encountered during polar scans of the bcc(110) surface. Referring to the $[1 \overline{1} 0]_{\mathrm{bcc}}$ direction as the $\phi_{\mathrm{bcc}}=0^{\circ}$ azimuth and to the $[001]_{\mathrm{bcc}}$ direction as the $\phi_{\mathrm{bcc}}=90^{\circ}$ one, the closest-packed atomic rows appear just along these azimuths, at $\theta=45^{\circ}(2.87 \AA$ interatomic spacing) and at $\theta=35.3^{\circ}$ (2.48 $\AA$ spacing), respectively. A remarkable similarity exists between these polar angles and those of the main features in the IAD's measured along the $[1 \overline{2} 1]_{\mathrm{fcc}}$ and $[1 \overline{1} 0]_{\mathrm{fcc}}$ azimuths, i.e., $\theta=47^{\circ}$ and $\theta=32^{\circ}$, respectively. Concerning the $[2 \overline{1} \overline{1}]_{\text {fcc }}$ azimuth data, the feature centered at $55^{\circ}$ undergoes a shape evolution as a function of film thickness, which is more complex, possibly related to which of the two orientation relationship occurs or to the presence of domains changing their relative weight. These arguments strongly suggest that a bcc(110) phase has formed above $3 \mathrm{ML}$. In the following, a quantitative analysis is presented, in order to characterize in detail the structural evolution of the $\mathrm{Fe}$ film in the medium-to-high coverage range and particularly to determine which in-plane orientation occurs at the bcc/fcc interface.

\section{Data modeling}

\section{Low coverage}

To determine the Fe atomic structure at low coverage, we measured $\mathrm{Fe} 2 p$ intensity patterns at low kinetic energy, over a large solid angle onto the 1.5-ML film. Photoelectrons were excited to a kinetic energy of $120 \mathrm{eV}$ with the twofold aim of enhancing both the backscattering effect over the forward one, due to the shape of the electron-atom scattering factor at low energy, and the surface sensitivity, due to the smaller IMFP of the photoelectrons.

The experimental pattern, shown in Fig. 4(a), displays threefold symmetry. The prominent feature is represented by a hexagon centered at the origin with inequivalent vertices starting at $\phi_{\mathrm{fcc}}=0^{\circ}$ and at $\theta$ between $40^{\circ}$ and $50^{\circ}$. Within the hexagon, a threefold high-intensity feature is evident at $\phi_{\mathrm{fcc}}=0^{\circ}, 120^{\circ}, 240^{\circ}$ and $\theta=32^{\circ}$, which forms, with the two hexagon sides nearby, an arrowlike shape. Close to the three other vertices, at $\phi_{\mathrm{fcc}}=60^{\circ}, 180^{\circ}, 300^{\circ}$ and $\theta=32^{\circ}$, weaker and smaller features appear. Outside the hexagon, dark regions at $\phi_{\mathrm{fcc}}=0^{\circ}, 120^{\circ}$, and $240^{\circ}$ are alternated with couples of bright bands, symmetric with respect to $\phi_{\mathrm{fcc}}=60^{\circ}, 180^{\circ}$, and $300^{\circ}$ azimuths.

The observed symmetry, appropriate for a fcc(111) surface, and the position of the threefold high-intensity feature within the hexagon, which closely matches the directions of first nearest-neighbor (NN) bonds in case of an fcc-site occupation, suggests that the film structure is pseudomorphic fcc(111), with fcc sites occupied at the interface. However, the possibility of an fcc(111) structure with mixed fcc-hcp site occupation and that of $\mathrm{bcc}(110)$ growth from the very 


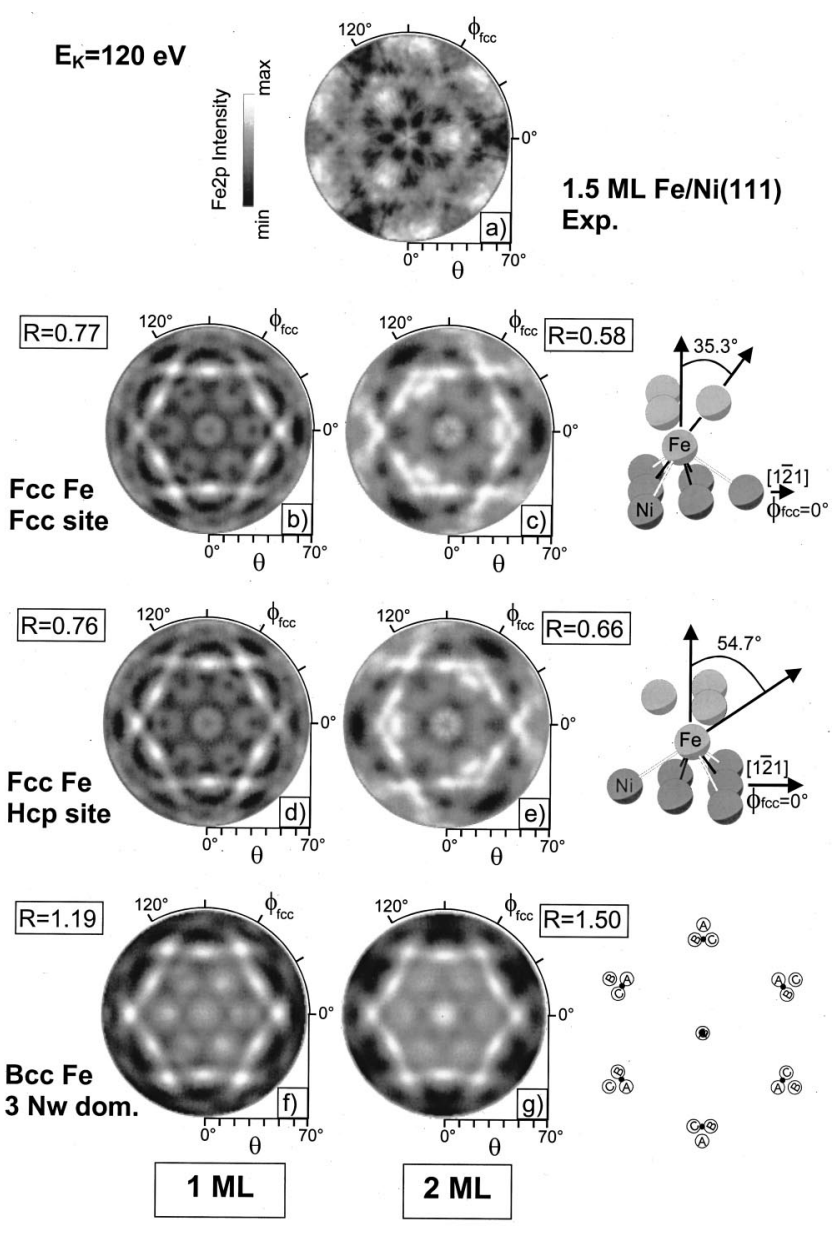

FIG. 4. (a) Stereographic projection of Fe $2 p$ intensity measured from a $1.5-\mathrm{ML} \mathrm{Fe} / \mathrm{Ni}(111)$ film at $E_{K}=120 \mathrm{eV}$. (b) and (c) $\mathrm{MS}$ calculations for 1- and 2-ML fcc Fe films, with Fe atoms at the interface occupying fcc hollow sites. (d) and (e) same as (b) and (c) but for Fe atoms occupying hcp hollow sites. (f) and (g) MS calculations for 1- and 2-ML bcc(110) Fe films: the patterns are linear combinations of the three equally populated NW domains. For each calculated pattern the $R$-factor value is displayed.

beginning (though, in this case, the expected symmetry should be either twofold for a single domain or sixfold for all the possible NW or KS domains occupied) have to be taken into account.

To clarify these points we performed calculations on 1and 2-ML-thick Fe clusters, with either fcc(111) or bcc(110) structures. For the fcc case, we considered two possible adsorption sites on the Ni surface, which are schematically represented in the stick and ball drawings: fcc hollow and hcp hollow. We notice that, if the fcc site is occupied, first NN's (black sticks) are at $\phi_{\mathrm{fcc}}=0^{\circ}, 120^{\circ}, 240^{\circ}$ and $\theta=35^{\circ}$ and second NN's (white sticks) are at $\phi_{\mathrm{fcc}}=60^{\circ}, 180^{\circ}, 300^{\circ}$ and $\theta=55^{\circ}$. If the hcp hollow site is occupied, first and second NN's exchange their $\phi_{\text {fcc }}$ coordinates. For bcc clusters, we considered both the case of three NW-oriented domains and that of six KS-oriented domains. Fe structural parameters were those of the $\mathrm{Ni}$ substrate, for fcc clusters, and those from best-fit parameters of the 6-ML film, for bcc clusters. For each calculated pattern, we report the values of two dif- ferent $R$ factors: $R$ is obtained by a combination of five different $R$ factors, as described in Ref. 25, $R_{P}$ is the more familiar Pendry $R$ factor. ${ }^{26}$

The calculation for the 1-ML fcc cluster, fcc site [Fig. 4(b)], shows a weak threefold symmetry, hardly distinguishable from a sixfold one. The pattern displays a hexagon with equivalent high-intensity sides but inequivalent vertices. They are located at $\theta=47^{\circ}$, but those at $\phi_{\mathrm{fcc}}=0^{\circ}, 120^{\circ}$, and $240^{\circ}$, corresponding to azimuths where first NN's are encountered, are slightly brighter than those at $\phi_{\mathrm{fcc}}=60^{\circ}$, $180^{\circ}$, and $300^{\circ}$, where second NN's are met. The visual agreement with the data $\left(R=0.77, R_{P}=0.082\right)$ is poor except for the hexagon shape, which is reproduced, but that in the data has a much more threefold symmetric character. The situation substantially improves $\left(R=0.58, R_{P}=0.052\right)$ when a second Fe layer is added [Fig. 4(c)]. Within the hexagon, bright features appear at $\theta=35^{\circ}$, close to the vertices at $\phi_{\mathrm{fcc}}=0^{\circ}, 120^{\circ}$, and $240^{\circ}$. These angular positions are those corresponding to first NN chains in case of an fcc-site occupation (see black sticks in the drawing beside). The fact that these peaks arise, on passing from one- to two-atom chains, might indicate that the forward scattering is not negligible even at these low energies. However, the same effect is not observed in the region corresponding to the second NN chains, which show an increased but diffuse intensity, so it is more likely that the bright peaks at first NN positions arise because of constructive interference effects related to path lengths. These features and the nearby hexagon sides produce bright arrowlike shapes, which agree fairly well with those observed in the data. On the contrary, the dark regions near the vertices at $\phi_{\mathrm{fcc}}=60^{\circ}, 180^{\circ}$, and $300^{\circ}$, do not reproduce very well what is experimentally observed. In the region outside the hexagon the agreement is satisfactory. Beyond the vertices at $\phi_{\mathrm{fcc}}=0^{\circ}, 120^{\circ}$, and $240^{\circ}$ there is a dark region, in good agreement with the data pattern, and, from the vertices at $\phi_{\mathrm{fcc}}=60^{\circ}, 180^{\circ}$, and $300^{\circ}$, two bright bands originate and diverge symmetrically. These bands, though more broadened, were also observed in the experimental pattern.

Passing to calculations for the fcc cluster, hcp site, it is clear how the patterns from 1- and 2-ML films [Figs. 4(d) and 4(e), respectively] are the same as for the fcc-site case, but rotated in azimuth by $60^{\circ}$. This accounts for the $60^{\circ}$ change, in the azimuthal position, which first and second NN bonds undergo on passing from one site to the other. The agreement with data, compared to the fcc-site case, remains the same for the 1-ML film $\left(R=0.76, R_{P}=0.085\right)$, and it is substantially worse for the 2-ML cluster $\left(R=0.66, R_{P}\right.$ $=0.098)$. One might interpret the weak-intensity features at $\phi_{\mathrm{fcc}}=60^{\circ}, 180^{\circ}, 300^{\circ}$ and $\theta=32^{\circ}$, in the experimental pattern, as a contribution from hcp-site domains, which show the "bright arrow" features there. We have considered the possibility of mixed fcc-hcp site domains (the so-called twinned fcc domains) by adding increasing amounts of the hcp-site pattern to the fcc-site one: the $R$-factor value was constantly increasing from that of pure fcc-site contribution.

We now consider the calculations for bcc(110) clusters. In Figs. 4(f) and 4(g), we present the results for 1- and 2-MLthick films with three NW-oriented domains, equally occu- 
pied. Single-domain patterns are not displayed because they yield twofold symmetry, in clear disagreement with the data. Both 1- and 2-ML patterns display a sharp hexagon similar to that produced by 1-ML fcc clusters. It is interesting to notice how the symmetry of the patterns changes. It is threefold for the 1-ML case, reflecting the symmetry of the substrate, and turns to the expected sixfold, when a second bcc layer is added. The agreement with data is not very satisfactory for both the 1-ML $\left(R=1.20, R_{P}=0.184\right)$ and the $2-\mathrm{ML}$ $\left(R=1.50, R_{P}=0.230\right)$ cases. The patterns from KS-oriented clusters, not shown here, are very similar to the NW ones, with just a slight broadening of all the bright features, due to the larger number of domains involved.

Finally, we have also considered the case of a 2-ML-thick film with a fcc, fcc site, interface layer, and either a NW- or KS-oriented bcc top layer. The calculated patterns, not shown, closely resemble those of the 2-ML-thick pure bcc phase. By assuming the bcc domains equipopulated, the following $R$ factors result for the NW case: $R=1.42, R_{P}$ $=0.219$. We also combined increasing percentages of these patterns to that of the 2-ML-thick fcc film, fcc site, but the resulting $R$ factors were increasingly higher than the best-fit ones.

\section{Medium-to-high coverage}

In this coverage range, we performed calculations for KSand NW-oriented bcc(110) clusters and compared them to the 6-, 9-, and 18-ML data. The multidomain character of both NW and KS orientations calls for an accurate structural model to be set up, which includes the correctly weighted averaging of all the possible azimuthal orientations of the bcc(110) domains, with respect to the inspected azimuths of the fcc(111) surface.

In principle, along each of the considered fcc azimuths, the NW orientation contributes with three nonequivalent azimuthal directions and the KS with six directions. However, due to the high symmetry of the chosen substrate azimuths, those bcc domains occupying mirror positions with respect to the fcc azimuth [see Fig. 3(b)] give an identical contribution to the measured intensity, i.e., the same bcc azimuths are aligned. In details, along the $[1 \overline{2} 1]_{\mathrm{fcc}}$ azimuth, the NW model aligns the $\phi_{\mathrm{bcc}}=0^{\circ}$ (domain $A$ ) and $60^{\circ}(B$ and $C$ ) azimuths and the KS model aligns the $\phi_{\mathrm{bcc}}=5^{\circ}$ (domains 2 and 3), $55^{\circ}$ ( 1 and 5), and $65^{\circ}$ (4 and 6) azimuths. Along the $[1 \overline{1} 0]_{\text {fcc }}$ azimuth the NW model contributes with the $\phi_{\text {bcc }}$ $=30^{\circ}(A$ and $B)$ and $90^{\circ}(C)$ azimuths, and the KS model contributes with the $\phi_{\mathrm{bcc}}=25^{\circ}$ ( 1 and 2), $35^{\circ}$ ( 3 and 4), and $85^{\circ}$ (5 and 6) azimuths. Finally, along the $[2 \overline{1} 1]_{\mathrm{fcc}}$ azimuth the NW and KS domains align exactly the same azimuths as for the $[1 \overline{2} 1]_{\text {fcc }}$ case but the couplings occur between different domains. We notice that the different shapes of the polar scans measured along the $[2 \overline{1} 1]_{\mathrm{fcc}}$ and $[1 \overline{2} 1]_{\mathrm{fcc}}$ azimuths yield evidence of a nonequal occupation of either KS or NW domains, so that a variable occupation of different domains has been included in the $R$-factor minimization procedure.

At a given coverage, we compared data and calculations for each fcc azimuth, minimizing the $R$ factor, $R$ (Ref. 25), upon variation of both structural parameters and domains

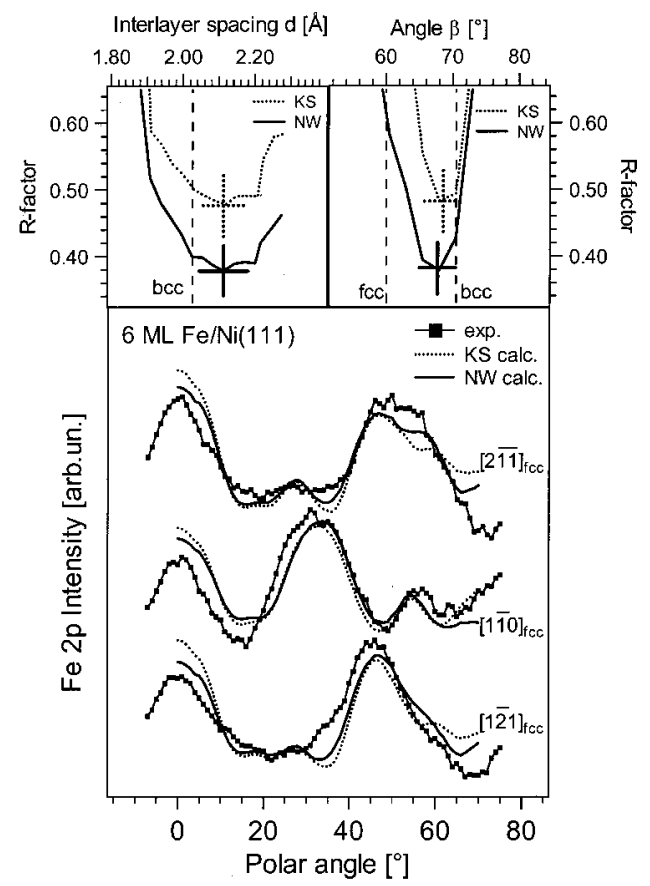

FIG. 5. Experimental Fe $2 p$ IAD's along the $[1 \overline{2} 1]_{\text {fcc }}$, $[1 \overline{1} 0]_{\mathrm{fcc}}$, and $[2 \overline{1} \overline{1}]_{\mathrm{fcc}}$ azimuths for the 6-ML Fe film compared to the best-fit calculations for KS and NW models. In the insets, the dependence of the $R$-factor vs fitting parameters is shown with horizontal and vertical error bars at the minimum.

occupation. We varied two structural parameters to account for the possible vertical and in-plane relaxations of the bcc $\mathrm{Fe}(110)$ lattice: the vertical interlayer spacing $d$ (varied simultaneously over the whole cluster, composed of five atomic layers) and the angle $\beta$ between the unit vectors of the surface cell [defined as in Fig. 3(a)]. The three sets of results were then averaged to obtain the overall best-fit result for that coverage.

In Figs. 5 and 6, the experimental IAD's measured at 6 and $9 \mathrm{ML}$ are shown along with the NW and KS best-fit calculations. In the top panels the $R$-factor behavior vs structural parameters is also shown. We observe that the overall agreement between calculations and data is satisfactory, and that all the main features in the experimental curves are well reproduced by the calculations. This confirms that the film has bcc(110) structure in the medium-to-high coverage range. Concerning the in-plane orientation model, at $6 \mathrm{ML}$ the $R$-factor curves clearly favor the NW model over the KS one, with a difference between the two minima being greater than the error. By inspection of the IAD curves, the best agreement of the NW model is evident, particularly along the $[1 \overline{2} 1]_{\mathrm{fcc}}$ and $[2 \overline{1} 1]_{\mathrm{fcc}}$ azimuths. The $R$-factor minima in the NW curves occur for an interlayer spacing $d_{\mathrm{NW}}=2.11 \AA$, corresponding to $\mathrm{a}+3.9 \%$ vertical expansion, and for an angle $\beta_{\mathrm{NW}}=67.7^{\circ}$, corresponding to a $-1.7 \%$ contraction of the surface cell area. At 9 ML a similar result occurs. The NW model gives best-fit results for a reduced interlayer spacing $d_{\mathrm{NW}}=2.08 \AA$, corresponding to a $+2.5 \%$ vertical expansion, and for a very similar $\beta$ angle $\left(68.0^{\circ}\right)$. Again, the best visual agreement of the NW model appears along the 


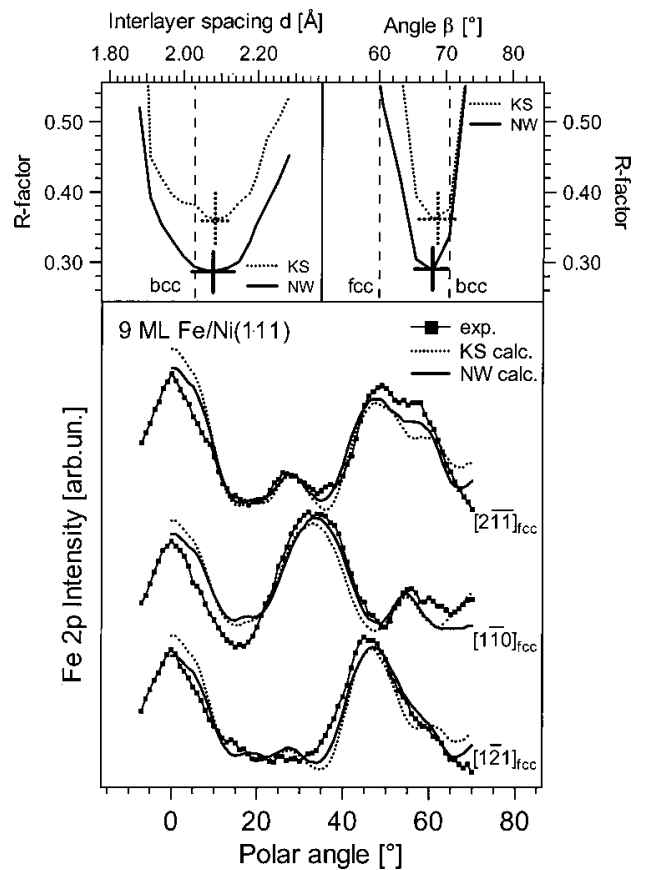

FIG. 6. Same as Fig. 5, but for the 9-ML Fe film.

aforementioned azimuths. For the 18-ML film, the difference between the NW and KS $R$-factor minima lies within the error, though the NW model still gives a lower absolute value. The best-fit interlayer spacing reduces to $2.05 \AA$ $(+1.0 \%)$ and the angle $\beta_{\mathrm{NW}}$ increases to $69.0^{\circ}(-1.0 \%$ of surface cell area), i.e., the two parameters are approaching equilibrium values for a bcc(110) cell. In Fig. 7, the behavior of the structural fitting parameters vs coverage is shown. Best-fit results are summarized in Table I. An error bar of $\pm 10 \%$ was estimated for the $R$-factor values, based on the statistical error affecting experimental data and assuming the calculations to be error-free. The error bar associated with the fitting parameters was calculated on the basis of the curvature of the $R$-factor curve at the minimum. ${ }^{27}$ We notice that a nonequal occupation of NW domains results, with domain $A$ having a $40 \%$ value and $B$ and $C$ both at $30 \%$. This is in agreement with the different shape of the experimental IAD's collected at the $[1 \overline{2} 1]_{\mathrm{fcc}}$ and $[2 \overline{1} \overline{1}]_{\mathrm{fcc}}$ azimuths, in the $40^{\circ}-$ $70^{\circ}$ region. The two features around $50^{\circ}$ and $60^{\circ}$ are the result of a superposition of two different contributions from the $\phi_{\mathrm{bcc}}=0^{\circ}$ and $\phi_{\mathrm{bcc}}=60^{\circ}$ azimuths. The calculations show that, for the $\phi_{\mathrm{bcc}}=0^{\circ}$ azimuth, a strong peak is present at $46^{\circ}$

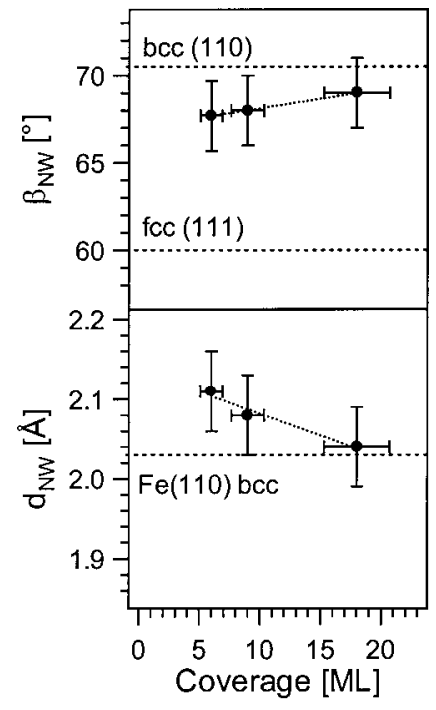

FIG. 7. Best-fit structural parameters, the interlayer distance $d_{\mathrm{NW}}$, and the angle $\beta_{\mathrm{NW}}$, as a function of the Fe coverage.

and, for the $\phi_{\mathrm{bcc}}=60^{\circ}$ one, a weaker peak at $58^{\circ}$ appears. Therefore, with reference to the $[1 \overline{2} 1]_{\text {fcc }}$ azimuth IAD's, the strong peak at $47^{\circ}$ is produced by the larger occupation of the domain $A$, contributing with the $\phi_{\mathrm{bcc}}=0^{\circ}$ azimuth, and the weak shoulder at $60^{\circ}$ belongs to the less occupied $B$ and $C$ domains, contributing with $\phi_{\mathrm{bcc}}=60^{\circ}$ azimuths. Along the $[2 \overline{1} \overline{1}]_{\mathrm{fcc}}$ azimuth, the enhancement of the $58^{\circ}$ component, reaching the same intensity as the $47^{\circ}$ one, is explained by the increased contribution from the $\phi_{\mathrm{bcc}}=60^{\circ}$ azimuths $(A$ and $C$ domains) and with a lower one from the $\phi_{\mathrm{bcc}}=0^{\circ}$ azimuth (domain $B$ ).

\section{DISCUSSION}

Measurements in the backscattering regime, combined with the MS calculations, suggest that the Fe structure is pseudomorphic fcc(111) up to $2 \mathrm{ML}$, with Fe atoms at the interface occupying fcc hollow sites. These indications come from both the visual and the $R$-factor based agreement between data and calculations. For one of the $R$ factors $(R)$ the value for the 2-ML fcc cluster, hcp site, is very close to that for the fcc site and, including $\pm 10 \%$ error bars, they partially overlap. However, as mentioned before, there was no improvement in the agreement by mixing fcc- and hcp-site domains. Our results are consistent with those of Theobald

TABLE I. Best-fit structural parameters and bcc domain occupations for the 6-, 9-, 18-ML Fe/Ni(111) films.

\begin{tabular}{cccccccc}
\hline \hline & & & & & \multicolumn{2}{c}{$\begin{array}{c}\text { NW domain } \\
\text { occupations (\%) }\end{array}$} \\
\cline { 6 - 8 } $\begin{array}{c}\text { Fe coverage } \\
(\mathrm{ML})\end{array}$ & $d_{\mathrm{NW}}(\AA)$ & $\beta_{\mathrm{NW}}(\mathrm{deg})$ & $\begin{array}{c}R_{\mathrm{NW}} \\
( \pm 10 \%)\end{array}$ & $\begin{array}{c}R_{\mathrm{KS}} \\
( \pm 10 \%)\end{array}$ & $A$ & $B$ & $C$ \\
\hline 6 & $2.11 \pm 0.06$ & $67.7 \pm 2.5$ & 0.38 & 0.48 & 43 & 28 & 29 \\
9 & $2.08 \pm 0.05$ & $68.0 \pm 2.5$ & 0.29 & 0.36 & 43 & 28 & 29 \\
18 & $2.05 \pm 0.03$ & $69.0 \pm 2.5$ & 0.28 & 0.32 & 40 & 25 & 35 \\
\hline \hline
\end{tabular}


et al. ${ }^{12}$ and with those of our previous work. ${ }^{19}$ Pseudomorphism up to similar coverages was also reported by Johnston, Arnold, and Venus ${ }^{13}$ and by Hoche and Elmers. ${ }^{14}$ A quantitative analysis of the data was attempted, but, in contrast to the forward-scattering case, poor dependence of the $R$ factor upon variation of the structural parameters was found. A possible explanation is that, at low kinetic energy, due to the scattering factor shape, the angular dependence of the scattering features becomes much broader than for the forwardscattering regime, and affects the sensitivity to small structural changes. Moreover, the quality of the experimental data in the backscattering regime is lower, being affected by a higher experimental error with respect to the forwardscattering data. Before discussing the results for the mediumto-high coverage range, a general consideration is necessary on the limitations imposed by the use of polar scans. Though the quantitative analysis was finally successful, the sensitivity to the in-plane bcc(110) cell orientation was influenced by the fact that the KS and NW models contribute with similar azimuthal directions (e.g., some differ by $\Delta \phi=5^{\circ}$ only) along the inspected fcc azimuths. It follows that, in the case that all the domains are present with similar occupations, the calculated IAD's tend to be similar, smearing out the differences between the two models. The use of azimuthal scans would probably give a higher sensitivity to this kind of analysis. However, at least for the 6- and 9-ML cases, the separation between the NW and KS $R$-factor minima is larger than the error bars, and a conclusion in favor of the NW model can be drawn on quantitative grounds.

The observed structural evolution, with a fcc-to-bcc transition developing at low coverage, fits the theoretical description by Bauer and van der Merwe ${ }^{9}$ of a bcc(110)/ fcc(111) interface, with an $r_{\mathrm{Fe} / \mathrm{Ni}}$ parameter and a weak layer/ substrate interaction energy (which is expected when film and substrate atomic species are similar ${ }^{28}$ ). In that case, for the first layer, pseudomorphism is predicted, independent of the $r$ value. Then, assuming that the elastic energy scales with the square root of the layer number, transition to a bcc(110) phase is predicted at low coverage. The same authors do not specify which in-plane orientation should occur at the bcc/fcc interface. In fact, $r_{\mathrm{Fe} / \mathrm{Ni}}$ lies in a critical region where neither NW nor KS energy curves have a minimum. However, the occurrence of a NW orientation has been reported by Fukuda and Gotoh ${ }^{10}$ with calculations on small clusters of $\mathrm{Fe}$ on a $\mathrm{Ni}(111)$ surface. The experimental study by Johnston and co-workers ${ }^{13}$ reports, at $12 \mathrm{ML}$, the copresence of both KS- and NW-oriented bcc(110) cells, with a value of the $\beta$ angle $\left(69.2^{\circ}\right)$ practically identical to ours. The present results extend the findings of our previous work, ${ }^{19}$ focused on the correlation between electronic and structural changes. The critical thickness of $4 \mathrm{ML}$ for the fcc-to-bcc transition, inferred from the appearance of extra spots in the LEED pattern, is basically confirmed by the present structural analysis, based on a quantitative method. Moreover, in that paper we assumed that the structural transition was based on a KS mechanism, on the basis of the angular separation between integer and extra spots. This appears contradicted by present results. As a matter of fact, the poor quality and misorientation of the LEED pattern shown in Ref. 19 does not allow for a discrimination between KS and NW orientations on the simple basis of the angular separation between integer and extra spots. Therefore, though the presence of the extra spots and, consequently, of an additional structural phase-which is likely to be bcc(110) - is not under question, the conclusion about the KS orientation of the bcc cell is highly questionable.

The trend of the vertical interlayer spacing vs thickness accounts for the angular shifts observed in the data, i.e., a reduction in the interlayer spacing implies an increase in the angular position of the off-normal features. We also notice that the best-fit values of the structural parameters are consistent with an elastic strain of the Fe volume cell, where the vertical expansion due to increased interlayer distances tends to be compensated for by a reduction of the surface cell area, as measured by the decrease of the $\beta$ angle. The behavior of the angular positions of the forward features, moving progressively to equilibrium values with thickness, suggests that the elastic strain release occurs in a smooth way rather than through abrupt mechanisms such as the formation of dislocations, which are reported for heteroepitaxial systems with sharp interfaces. ${ }^{29}$ However, it cannot be excluded that within the probed area, few regions undergo this sudden change and the others remain strained, so that the averaged result is the one observed. Concerning the values of the bcc lattice parameters, which are out of equilibrium even for a relatively high thickness (18 ML), the concentration of carbon in the films has to be taken into account. Quinn et al. ${ }^{30}$ observed a 3\% expansion of the vertical interlayer spacing and equilibrium in-plane parameters for a 200-ML-thick bcc Fe film, grown on $\operatorname{Pd}(001)$. By invoking the formation of dislocations, they explained the in-plane strain release, but the out-of-plane strain remained unexplained. They attributed the tetragonal distortion to the high carbon concentration (22 at. \%, $5.7 \mathrm{wt} \%)$ in the film, based on the value of carbon concentration $(0.65 \mathrm{wt} \%)$ sufficient to cause $3 \%$ tetragonality in martensite. The present case seems different for two reasons: (i) the vertical expansion we observe is sensibly lower $(1 \%)$ and we still observe an in-plane contraction which, according to elasticity, can justify it, (ii) the carbon concentration in our films (9 at. \%, i.e., $2 \mathrm{wt} \%$ ) is much lower than that reported by Quinn et al., though, according to the case of martensite, it could be high enough to contribute to a tetragonal distortion.

\section{CONCLUSIONS}

A detailed structural study of $\mathrm{Fe}$ ultrathin films, grown on a $\mathrm{Ni}(111)$ single crystal at room temperature, has been carried out over a wide coverage range, employing anglescanned photoelectron diffraction and a MS calculation modeling of the data. The use of synchrotron radiation has allowed us to exploit both backscattering and forwardscattering energy regimes, to enhance the structural sensitivity at lower and higher film thicknesses, respectively. From the results in the backscattering regime, we have indications that, within a 2-ML coverage, the Fe film has a fcc(111) pseudomorphic structure, with interfacial atoms occupying fcc hollow sites, in agreement with previous results. ${ }^{12}$ In- 
creasing the coverage, from $3 \mathrm{ML}$ onwards, a transition to a bcc(110) phase was observed and investigated in the forward-scattering regime. Quantitative analysis was applied to 6-, 9-, and 18-ML data, minimizing a combination of $R$ factors as a function of the in-plane orientation relationship (KS or NW) and of two structural parameters. Their choice accounts for the vertical and in-plane relaxations, i.e., the vertical interlayer distance between bcc(110) planes $(d)$ and the angle between surface basis vectors $(\beta)$. We find that the NW in-plane orientation is favored over the KS one and develops with three nonequally populated domains. Between 6 and $18 \mathrm{ML}, d_{\mathrm{NW}}$ relaxes from $2.11 \AA(+3.9 \%$ expansion $)$ to $2.05 \AA(+1.0 \%)$, respectively, in agreement with the an- gular shift observed for the forward-focusing features. In the same coverage range $\beta_{\mathrm{NW}}$ slightly increases from $67.7^{\circ}$ to $69.0^{\circ}$, corresponding to a contraction of surface cell area by $-1.7 \%$ and $-1.0 \%$, respectively. Finally, concerning the growth mode at low coverage, indications of good wetting and of a sharp $\mathrm{Fe} / \mathrm{Ni}$ interface were found.

\section{ACKNOWLEDGMENTS}

The authors wish to acknowledge L. Marassi and the ALOISA team for assistance during the experiment, and O. Moze for a critical reading of the manuscript. Financial support by INFM and MURST are also acknowledged.

${ }^{17}$ S. D’Addato, L. Marassi, P. Luches, G. C. Gazzadi, L. Pasquali, R. Verucchi, P. Finetti, and S. Nannarone, Surf. Sci. 487, 258 (2001).

${ }^{18}$ G. B. Fratucello, A. M. Prandini, P. Luches, S. D’Addato, A. J. Patchett, and S. Nannarone, J. Magn. Magn. Mater. 210, 349 (2000).

${ }^{19}$ S. D’Addato, L. Pasquali, G. C. Gazzadi, R. Verucchi, R. Capelli, and S. Nannarone, Surf. Sci. 454-456, 692 (2000).

${ }^{20}$ C. S. Fadley, in Synchrotron Radiation Research: Advances in Surface and Interface Science, edited by R. Z. Bachrach (Plenum, New York, 1992); W. J. Egelhoff, Jr., in Ultrathin Magnetic Structures, edited by J. A. C. Bland and B. Heinrich (Springer-Verlag, Berlin, 1994).

${ }^{21}$ L. Floreano, G. Naletto, D. Cvetko, R. Gotter, M. Malvezzi, L. Marassi, A. Morgante, A. Santaniello, A. Verdini, F. Tommasini, and G. Tondello, Rev. Sci. Instrum. 70, 3855 (1999). Beam line website: http://tasc.area.trieste.it/tasc/lds/aloisa/ aloisa.html

${ }^{22}$ S. Tanuma, C. J. Powell, and D. R. Penn, Surf. Interface Anal. 17, 911 (1991).

${ }^{23}$ Y. Chen, F. J. G. de-Abajo, A. Chasse, R. X. Ynzunza, A. P. Kaduwela, M. A. Van-Hove, and C. S. Fadley, Phys. Rev. B 58, 13121 (1998). See website http://electron.lbl.gov/mscdpack/

${ }^{24}$ V. L. Moruzzi, J. F. Janak, and A. R. Williams, Calculated Electronic Properties of Metals (Pergamon, New York, 1978).

${ }^{25}$ R. S. Saiki, A. P. Kaduwela, M. Sagurton, J. Osterwalder, D. J. Friedman, C. S. Fadley, and C. R. Brundle, Surf. Sci. 282, 33 (1993).

${ }^{26}$ J. B. Pendry, J. Phys. C 13, 937 (1980).

${ }^{27}$ H. B. Nielsen and D. L. Adams, J. Phys. C 15, 615 (1982).

${ }^{28}$ E. Bauer, Appl. Surf. Sci. 11/12, 479 (1982).

${ }^{29}$ C. Günther, J. Vrijmoeth, R. Q. Hwang, and R. J. Behm, Phys. Rev. Lett. 74, 754 (1995); E. Lundgren, B. Stanka, M. Schmid, and P. Varga, Phys. Rev. B 62, 2843 (2000).

${ }^{30}$ J. Quinn, Y. S. Li, H. Li, D. Tian, F. Jona, and P. M. Marcus, Phys. Rev. B 43, 3959 (1991). 